\title{
A Life-Threatening Presentation of Primary Small Cell Colorectal Non-Hodgkin Lymphoma as Cardiac Tamponade and Its Challenging Management
}

\author{
Amr Elkammash ${ }^{1}$, Mohamed Ayman Abdel-Hay ${ }^{2}$, Saleh Kanaan ${ }^{1}$, Mustafa Alsinan ${ }^{1}$, Yosra Taha ${ }^{1}$, Mohammed Fadul ${ }^{1}$, Nourhan Degheidy ${ }^{3}$ \\ ${ }^{1}$ Department of General Internal Medicine, University Hospitals Dorset, The Royal Bournemouth Hospital, Bournemouth, UK \\ ${ }^{2}$ Department of Cardiology, Alexandria University Hospitals, Alexandria, Egypt \\ ${ }^{3}$ Medical Research Institute, Alexandria, Egypt
}

Received: 06/07/2021

Accepted: $12 / 07 / 2021$

Published: 06/08/2021

How to cite this article: Elkammash A, Abdel-Hay MA, Kanaan S, Alsinan M, Taha Y, Fadul M, Degheidy N. A life-threatening presentation of primary small cell colorectal non-hodgkin lymphoma as cardiac tamponade and its challenging management. EJCRIM 2021;8: doi:10.12890/2021_002753.

Conflicts of Interests: The authors declare there are no competing interests.

Acknowledgements: Our team would like to thank Ms Michele Berner for proofreading the report.

This article is licensed under a Commons Attribution Non-Commercial 4.0 License

\section{ABSTRACT}

Colorectal non-Hodgkin lymphoma (NHL) is quite aggressive and rare, only constituting less than $1 \%$ of all cases of colorectal cancer. The pericardium is an extremely rare first site of metastasis. Cardiac tamponade can be a life-threatening initial presentation. We report a 55-year-old female who presented with severe shortness of breath, intermittent abdominal pain and altered bowel habits. She had low blood pressure with congested neck veins. Her echocardiogram showed pericardial and cardiac infiltration with tumour mass; a large pericardial effusion with signs of cardiac tamponade. There was no safe window for percutaneous drainage, and the patient was not physically fit for surgical drainage. A multidisciplinary approach was used to diagnose and manage the case involving a cardiologist, gastroenterologist, pathologist, radiologist and oncologist. CT scans of the whole body showed a large rectosigmoid mass infiltrating the uterus and adnexa. Flexible sigmoidoscopy showed a large bleeding mass at the rectosigmoid junction. The biopsy confirmed small cell NHL. Three cycles of chemotherapy were urgently commenced over a period of 5 weeks ( 1 cycle of CVP; 2 cycles of CHOP). The patient showed significant symptomatic improvement. A 5-week follow-up echocardiogram showed significant shrinkage of the pericardial tumour and only a small rim of pericardial effusion. The effusion did not recollect in her follow-up echocardiograms. A year later, she was referred to the palliative care team due to the further spreading of her lymphoma. In conclusion, colorectal small cell NHL may initially present as cardiac tamponade. Urgent initiation of chemotherapy can be a treatment option whenever a drainage procedure is unsafe.

\section{LEARNING POINTS}

- Colorectal small cell NHL is a quite rare malignancy that may present initially with pericardial metastasis.

- Cardiac tamponade secondary to colorectal NHL is a life-threatening presentation. It can be managed by timely chemotherapy alone whenever the usual drainage procedures are not safe.

- A multidisciplinary approach is a cornerstone in the management of unstable lymphoma patients. It helps the rapid diagnosis and initiation of appropriate chemotherapy.

\section{KEYWORDS}

Tamponade, Iymphoma, chemotherapy, drainage, colon 


\section{INTRODUCTION}

Colorectal primary non-Hodgkin lymphoma ( $\mathrm{NHL}$ ) is quite rare. It accounts for approximately $1.4 \%$ of all cases of $\mathrm{NHL}$ and less than $1 \%$ of all colorectal malignancies ${ }^{[1]}$. The small cell type was found to be the least common in colorectal $\mathrm{NHL}$, with a prevalence of approximately $6 \%$ in a case series ${ }^{[2]}$. Cardiac tamponade occurs when pericardial effusion accumulates fast and compresses the cardiac chambers. Urgent drainage is the usual treatment of such cases through either a percutaneous or surgical approach ${ }^{[3]}$. Patients with malignant effusion can be treated with systemic chemotherapy alone ${ }^{[4]}$. There are no reported cases in the literature of cardiac tamponade secondary to small cell colorectal NHL that were treated with systemic chemotherapy alone.

\section{CASE DESCRIPTION}

We describe a 55-year-old female who presented a 7-day history of rapidly progressive shortness of breath. She had vague, diffuse abdominal pain, unexplained weight loss and difficulty in micturition for a few weeks before presentation. On examination, the patient had a low BMI, congested neck veins and increased work of breathing. Her blood pressure was $100 / 60 \mathrm{mmHg}$, and her pulse was 110 beats per minute. She did not have any signs of systemic hypoperfusion, with good urine output and normal lactate levels in the venous blood gas analysis. Heart sounds were distant, and the lungs were clear on auscultation. Abdominal examination revealed a hard fixed pelvic abdominal mass in the left iliac fossa but no other palpable lymph nodes. The ECG had low QRS voltage but did not show any signs of pericarditis. The chest $x$-ray showed an increased cardiothoracic ratio, suggestive of the presence of pericardial effusion. An urgent echocardiogram revealed that the pericardium was infiltrated with a shaggy tumour mass of variable echogenicity. The mass also infiltrated the walls of the right atrium (RA) and right ventricle (RV), appearing inside their cavities. In addition, there was a large pericardial effusion with diastolic RV free wall collapse, signifying cardiac tamponade (Fig. 1).

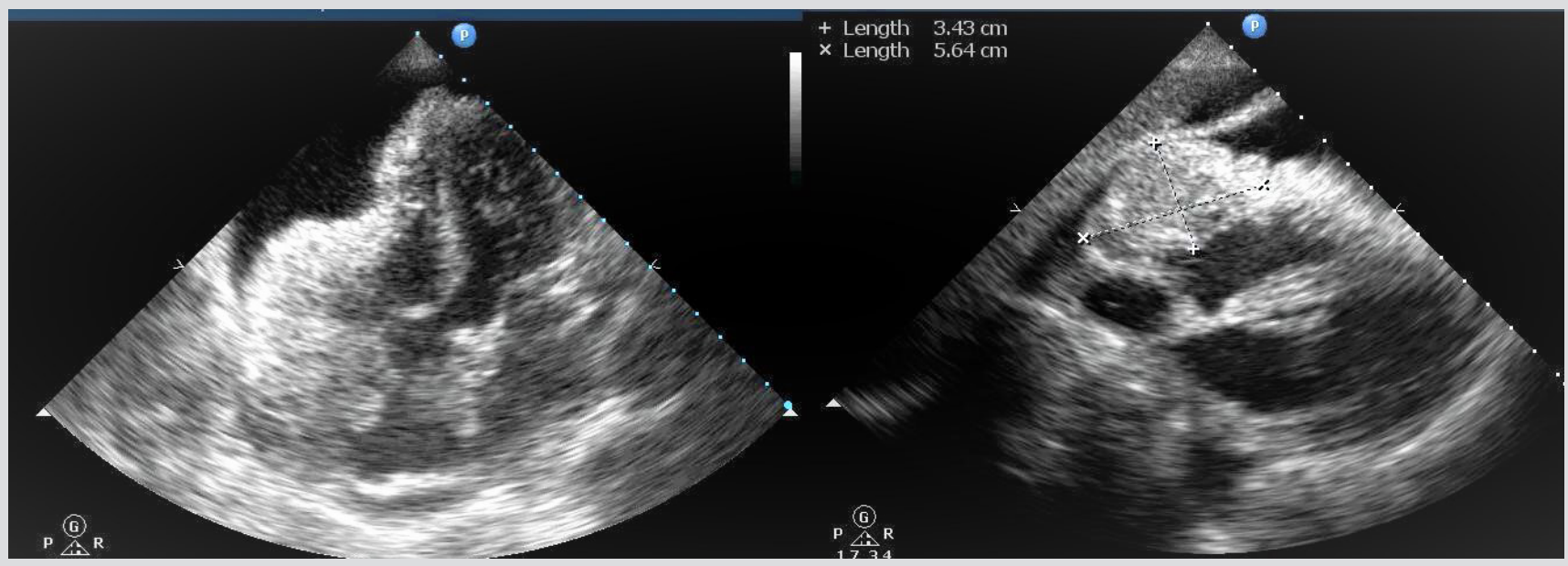

Figure 1. Transthoracic echocardiogram. Left: apical 4 chamber view showing tumour infiltration of the pericardium, right atrial and right ventricular walls, large pericardial effusion with diastolic collapse of the right ventricular free wall. Right: subcostal view showing the interposition of the tumour mass into the window for pericardiocentesis

The tumour mass interposed the needle track for pericardiocentesis, which made it unsafe for the patient. The patient's general condition was unfavourable to have general anaesthesia for surgical drainage. The patient was admitted to the CCU, resuscitated with IV fluids, which improved her blood pressure. To determine the primary source of the pericardial tumour, a whole-body CT scan was conducted. It illustrated the presence of a sizable pelviabdominal soft tissue lesion, averaging $13 \mathrm{~cm}$ in diameter, with heterogeneous enhancement and areas of cystic necrosis. The lesion was inseparable from the adnexa and uterus; the rectosigmoid segment showed concentric mural thickening (Fig. 2). The case was discussed in a multidisciplinary meeting involving the cardiologist, gastroenterologist, pathologist, radiologist, haematologist and surgeon. The tumour was not suitable for surgical removal, and the team decided to go for the chemotherapy option. An urgent flexible sigmoidoscopy showed the presence of a large mass narrowing the rectosigmoid junction with excessive bleeding (Fig. 3). A biopsy from the mass showed diffuse submucosal infiltration with a mixture of small and large cells, showing some cleaved forms. Immunostaining was positive for leucocyte common antigen and negative for chromogranin; this indicated colonic NHL of small cell type.

We referred the patient urgently to the medical oncology department, where she received 3 cycles of chemotherapy. The first cycle of CVP (cyclophosphamide, vincristine and prednisone) was given within 3 days of initial presentation. A few days later, the patient felt an 
improvement of symptoms. A follow-up echocardiogram was obtained every 3 days. That showed gradual shrinkage of the tumour size and a reduction in the amount of pericardial effusion. Three weeks later, she received a second cycle of CHOP (cyclophosphamide, doxorubicin, vincristine and prednisone). That was followed by a third cycle of CHOP in 2 weeks.

The tumour mass had shrunk significantly on the echocardiogram obtained a week later, with only a residual small rim of pericardial effusion (Fig. 4). The patient's symptoms resolved completely. The effusion did not reaccumulate in her follow-up echocardiograms. She continued her chemotherapy for 12 months; however, she was referred later to the palliative care team due to further spreading of her lymphoma.

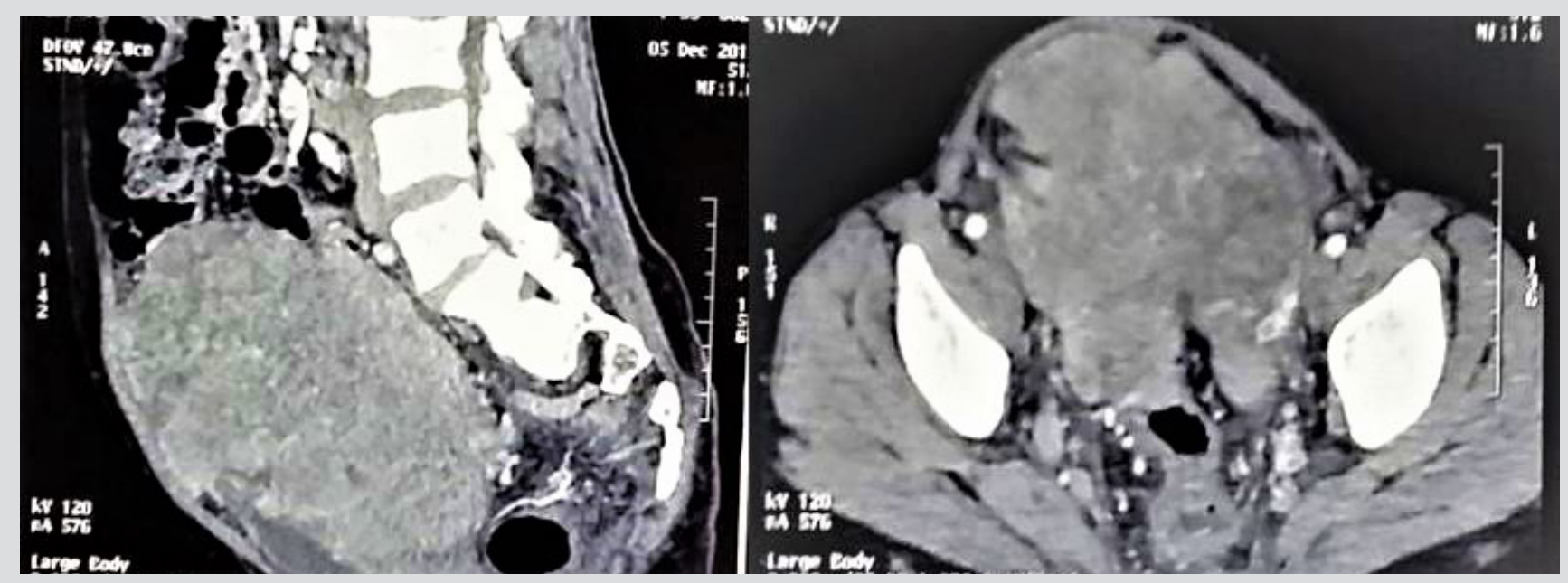

Figure 2. CT of the abdomen and pelvis with IV contrast, showing a heterogenous pelviabdominal soft tissue mass. Left: sagittal view. Right: transverse view
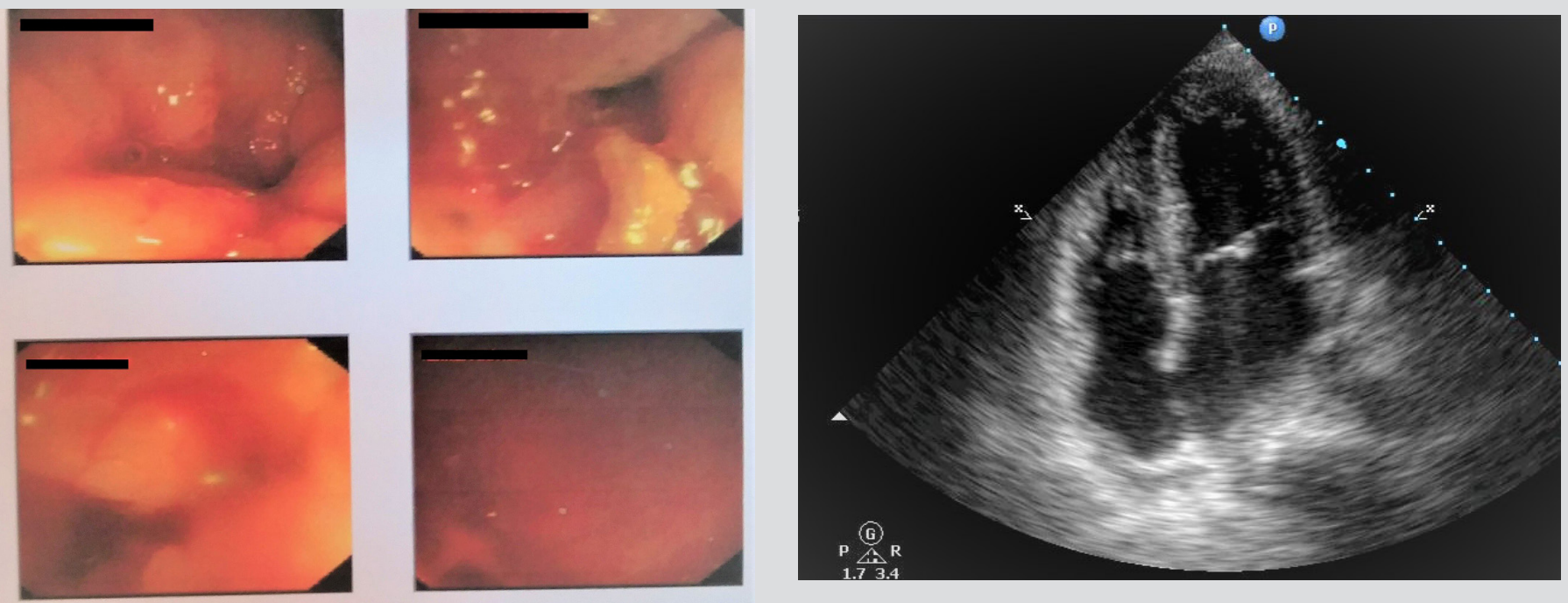

Figure 3. Pictures from the flexible sigmoidoscopy of the patient showed a lobulated rectosigmoid mass narrowing the lumen

Figure 4. Transthoracic echocardiogram after the third cycle of chemotherapy; it shows marked shrinkage of the pericardial tumour mass, clearance of the RA and RV walls and a mild rim of pericardial effusion

\section{DISCUSSION}

This report presents an uncommon presentation of a rare type of colorectal malignancy; small cell NHL. It also demonstrates a non-invasive treatment option for cardiac tamponade caused by this malignancy, which can be used whenever other drainage options are deemed difficult. The incidence of secondary pericardial effusion in $\mathrm{NHL}$ patients has been reported to be as low as $0.8 \%{ }^{[5]}$. Cardiac tamponade is a lifethreatening complication of pericardial metastasis with a high mortality rate. The success rate of treatment of malignant pericardial effusion using systemic chemotherapy alone was limited compared to that combined with a drainage procedure. The success of chemotherapy alone 
was higher in breast cancer and lymphoma patients, as these tumours are more chemotherapy-sensitive. Percutaneous pericardiocentesis needs a safe free space between the visceral and parietal layers of the pericardium without interposing masses. Surgical drainage, with the formation of a window between the pericardium and the pleura, needs a physically fit patient for general anaesthesia ${ }^{[4]}$. Both conditions were not found in our patient, which left us with the chemotherapy alone option. The case illustrates a practical example of the usefulness of the systemic chemotherapy-alone approach, even in life-threatening situations. Our literature review could not identify any other reports on the use of chemotherapy alone in the management of cardiac tamponade secondary to primary extranodal small cell NHL.

\section{CONCLUSION}

Extranodal lymphomas can initially present with systemic spread. Gastrointestinal lymphomas should be considered among the causes of malignant pericardial effusion despite their rarity. A multidisciplinary team approach helps in reaching the diagnosis and starting chemotherapy fast. Chemotherapy is effective in the treatment of pericardial effusions secondary to small cell colorectal NHL; however, the long-term prognosis remains unfavourable.

\section{REFERENCES}

Quayle FJ, Lowney JK. Colorectal lymphoma. Clin Colon Rectal Surg 2006;19:49-53.

Bairey O, Ruchlemer R, Shpilberg O. Non-Hodgkin's lymphomas of the colon. Isr Med Assoc J 2006;8:832-835.

Bodson L, Bouferrache K, Vieillard-Baron A. Cardiac tamponade. Curr Opin Crit Care 2011;17:416-424.

Çelik S, Lestuzzi C, Cervesato E, Dequanter D, Piotti P, De Biasio M, et al. Systemic chemotherapy in combination with pericardial window has better outcomes in malignant pericardial effusions. J Thorac Cardiovasc Surg 2014;148:2288-2293.

5. Das DK. Serous effusions in malignant lymphomas: a review. Diagn Cytopathol 2006;34:335-347. 